\title{
Traduire
}

Revue française de la traduction

229 | 2013

L'environnement, une spécialisation durable?

\section{Une spécialisation, le domaine de l'environnement}

\section{Abigail Dahlberg}

\section{(2) OpenEdition}

\section{Journals}

Édition électronique

URL : http://journals.openedition.org/traduire/578

DOI : $10.4000 /$ traduire. 578

ISSN : 2272-9992

Éditeur

Société française des traducteurs

Édition imprimée

Date de publication : 15 décembre 2013

Pagination : 16-25

ISSN : 0395-773X

\section{Référence électronique}

Abigail Dahlberg, «Une spécialisation, le domaine de l'environnement », Traduire [En ligne], 229 | 2013,

mis en ligne le 15 décembre 2015, consulté le 19 avril 2019. URL : http://journals.openedition.org/ traduire/578 ; DOI : 10.4000/traduire.578 


\section{Une spécialisation, le domaine de l'environnement}

\section{Abigail Dahlberg}

Les traducteurs ayant choisi de se spécialiser dans un domaine qui les intéresse, voire les passionne, gagnent certainement en efficacité. S'agissant de textes liés aux problèmes écologiques, ce choix prend une dimension supplémentaire car le traducteur peut alors avoir un impact direct sur son environnement. Les problématiques liées à l'environnement connaissent actuellement une forte croissance sur le marché de la traduction, ce qui s'explique par un durcissement des lois et une accélération de la prise de conscience face aux dégâts provoqués par l'activité humaine. Nous examinons ici les raisons du développement rapide de ce domaine sur le marché de la traduction, ainsi que les possibilités et les défis qui s'offrent aux traducteurs choisissant cette spécialité. Nous proposons également, à toutes fins utiles, quelques informations sur les ressources disponibles pour les traducteurs.

\section{Une planète en mutation}

Le terme " environnement " évoque nombre de problématiques : pollution de l'air, changement climatique, émissions diverses, conservation des sols, catastrophes environnementales (inondations et marées noires, par exemple), politique et technologie environnementales, protection de la nature, traitement de l'eau et des eaux usées, gestion des déchets, etc.

Les gouvernements travaillent désormais de concert pour s'atteler à la protection de l'environnement et aux mesures nécessaires pour réparer les dégâts accumulés au fil des années. La Convention-cadre des Nations Unies sur les changements climatiques (CCNUCC) et ses mécanismes ont dynamisé la recherche de solutions destinées à atténuer les changements climatiques. En 1997, les parties à la CCNUCC ont adopté le Protocole de Kyoto qui impose aux pays développés de réduire leurs émissions de gaz à effet de serre. Pour la première période d'engagement, de 2008 à 2012, 37 pays développés et 15 pays membres de l'UE ont accepté de réduire en moyenne leurs émissions de gaz à effet de serre de $5 \%$ par rapport aux niveaux de 1990. Parmi les exceptions notables à la liste des 191 pays ayant ratifié le Protocole de Kyoto, citons les États-Unis et le Canada, qui se sont retirés du Protocole l'an dernier. 
Le Protocole de Kyoto a été amendé en 2012 afin de préparer la deuxième période d'engagement (2013-2020), pour laquelle 37 pays devront s'efforcer de respecter les objectifs fixés. Les pays en développement n'ont pas d'obligations en termes d'émissions de gaz à effet de serre, mais ils s'engagent à les réduire et peuvent participer au Mécanisme pour un développement propre (MDP). Le MDP permet aux pays développés d'investir dans des projets destinés à réduire les émissions de gaz à effet de serre dans les pays en développement comme alternative à des dépenses beaucoup plus conséquentes en vue de réduire ces émissions chez eux. En juillet 2013, le MDP enregistrait son 7 000e projet. La CCNUCC indique que le MDP a suscité plus de 215 milliards de dollars US d'investissements pour un développement économique à faible émission de carbone dans les pays en développement au cours de la dernière décennie.

II ne faut pas sous-estimer les besoins en traduction générés par ces projets. En effet, les projets bilatéraux ou multilatéraux de ce type entre pays industrialisés et pays en développement produisent nombre de documents.

\section{Un marché en expansion}

Voyons plus précisément ce qui alimente la croissance du marché de la traduction spécialisée dans ce domaine et dans quelle mesure cette dernière peut assurer un revenu suffisant aux traducteurs qui s'y consacrent. Précisons qu'à la différence des domaines financier, médical ou juridique, l'environnement, domaine passionnant, présente encore une certaine pénurie de traducteurs qualifiés spécialisés. On peut donc parler d'un marché porteur.

La législation et les politiques environnementales constituent les premières sources de documents. En effet, après la conclusion d'accords internationaux tel le Protocole de Kyoto, les gouvernements des pays signataires doivent élaborer les projets de directives et de lois nécessaires à l'application des règles environnementales. Pour ce faire, ils peuvent être amenés à s'inspirer des choix opérés par d'autres pays, suscitant ainsi le besoin de traductions. Au bout de la chaîne, les autorités locales et régionales chargées de l'environnement doivent également prendre les dispositions ad hoc. Les pays accueillant un grand nombre d'immigrés ou possédant plusieurs langues officielles doivent traduire tous les documents destinés au public. De même, les initiatives régionales comme les directives et réglementations de l'Union européenne doivent être traduites en plusieurs langues.

Pour atteindre les objectifs nationaux fixés, entreprises et municipalités investissent dans les technologies de l'environnement. Les anglophones pourront trouver là une source de textes à traduire car les entreprises européennes ont acquis une grande expertise dans la mise au point de technologies de pointe susceptibles d'exportation vers le marché américain. D'autre part, l'opposition croissante du public aux projets de création ou d'expansion de décharges publiques conduit les autorités locales à étudier d'autres solutions, dont les possibilités de recyclage, d'autant plus intéressantes que les prix des produits recyclés sont relativement élevés. 
Mondialisation oblige, nombre de grandes entreprises du secteur environnemental souhaitent proposer des versions de leur site internet et de leurs communiqués de presse en différentes langues. Dans ce secteur, les rapports sur la responsabilité sociale des entreprises sont très en vogue, et d'innombrables entreprises (même si elles ne sont pas directement impliquées dans le secteur environnemental) précisent les dispositions prises pour réduire leur impact sur l'environnement. Ces rapports présentent généralement les chiffres concernant l'utilisation de matériaux recyclés, les économies d'énergie et la gestion de l'eau. Nombre d'entreprises font également certifier leurs usines et leurs sites selon les normes ISO internationales, plus particulièrement la série ISO 14000 traitant des normes de gestion environnementale, destinées à aider les entreprises à minimiser les effets négatifs de leur fonctionnement sur l'environnement.

Si vous envisagez de vous spécialiser dans l'environnement, votre paire de langues est un facteur clé. Dans mon cas, l'Allemagne étant un acteur reconnu du secteur de la gestion des déchets et du recyclage, je sais que je peux compter sur un volume de travail suffisant. Pour les traducteurs ayant le français parmi leurs langues de travail, il existe une niche qui pourrait se révéler très intéressante, étant donné les besoins croissants d'eau potable en Afrique du Nord : celle du traitement des eaux usées et du dessalement de l'eau de mer. D'autres secteurs ont également des besoins en traduction : pensez aux énergies renouvelables et à la construction HQE (Haute Qualité Environnementale).

\section{Les défis}

Revers de la médaille, les traducteurs du secteur environnemental rencontrent des difficultés similaires à celles présentes sur d'autres marchés de niche. En effet, cette spécialisation regroupe une vaste gamme de textes à traduire, du simple dépliant informant les foyers des nouvelles règles de tri des déchets jusqu'aux accords internationaux sur l'environnement. Les traducteurs spécialisés doivent donc maîtriser divers registres et traiter des textes de genres très divers, notamment des rapports annuels, communiqués de presse, descriptifs techniques, rapports de recherche et présentations destinées à des conférences. En effet, si vous décidez de vous spécialiser sur un marché de niche, vous devez offrir à vos clients un service répondant à tous leurs besoins de traduction pour la spécialité concernée. Les traducteurs se sont également heurtés à l'insuffisance des formations disponibles en la matière ; heureusement, les webinaires ont permis de pallier ce manque et il est désormais possible de suivre des cours en ligne sans quitter son bureau. Les traducteurs spécialisés peuvent aussi se tourner vers les formations destinées aux professionnels du secteur de l'environnement, bonne façon de rester à jour dans ses connaissances. Le plus souvent, le traducteur aura recours à l'auto-formation, indispensable en raison de l'évolution rapide du domaine.

Le traducteur spécialisé dans l'environnement a droit à son lot de terminologie bizarre et surprenante. En outre, la terminologie ou les concepts peuvent exister dans un pays et pas 
encore dans un autre. Ainsi, en matière de recyclage des déchets, nombre de pays d'Europe occidentale ont des années-lumière d'avance sur les États-Unis. Beaucoup de bureaux en Allemagne disposent d'au moins six poubelles différentes pour chaque catégorie de déchets : papier, plastique, métal, verre, déchets organiques et déchets résiduels (tout le reste). Pour limiter les opérations de ramassage, une municipalité a envisagé la création d'une poubelle associant la poubelle grise (déchets résiduels) et la jaune (emballages légers) sous la forme d'une poubelle à rayures grises et jaunes (dite Zebratonne en allemand). Ici, une traduction littérale aurait pu laisser perplexe le lecteur anglophone, la description de l'opération était donc plus parlante. Voilà un exemple des stratégies utilisées par le traducteur spécialisé dans le cadre de la mise en œuvre d'une politique de l'environnement.

\section{Ressources}

L'un des problèmes principaux auxquels est confronté le traducteur est le manque de dictionnaires bilingues spécialisés dans ce domaine. II existe peu de dictionnaires papier et ils sont souvent obsolètes puisqu'ils ont une dizaine d'années. Pour choisir le bon ouvrage, le traducteur spécialisé doit le tester en cherchant un mot qui pose un problème de traduction tout en étant suffisamment courant pour être répertorié. Par exemple en allemand, je cherche Altlastensanierung, que l'on peut traduire par "décontamination des sites pollués(1) " [cleanup of brownfield sites en anglais américain ou remediation of contaminated sites en anglais britannique]. Si je trouve une traduction littérale du type old site sanitation (" nettoyage d'un site ancien "), le dictionnaire est disqualifié. Ce manque de dictionnaires bilingues ou multilingues incite certains traducteurs à envisager de créer et publier leurs propres dictionnaires, et seul le manque de temps et de financement les empêchent de passer à l'acte.

La bonne nouvelle, c'est la multitude de ressources monolingues. National Geographic a publié de nombreux livres pertinents sur les questions de l'environnement. Les traducteurs exerçant en Europe disposent avec Ends Europe Daily d'une ressource précieuse couvrant les dernières évolutions des politiques nationales sur l'environnement. Autre possibilité intéressante, l'abonnement aux lettres d'information publiées par les associations environnementales et les organisations non gouvernementales, excellentes sources de terminologie. Indispensable enfin, s'abonner aux revues spécialisées en langues source et cible.

\section{Revues professionnelles}

J'ai commencé ma carrière comme traductrice salariée et journaliste dans une entreprise allemande publiant des revues spécialisées pour divers secteurs. Près de dix ans plus tard, cet éditeur est resté mon meilleur client. Cette relation m'a permis de vérifier concrètement les multiples avantages que peut apporter la consultation régulière de ce type de revues.

(1) Traduction proposée par le Wörterbuch der industriellen Technik, Deutsch-Französisch, de Richard Ernst (Oscar Brandstetter Verlag). 


\section{Conseils pour sélectionner des abonnements}

Une simple recherche via Google ne fournira pas nécessairement les meilleurs résultats, favorisant l'entreprise qui aura fait le plus de publicité. Lancez plutôt une discussion sur un groupe Linkedln dans votre spécialité ou interrogez vos clients pour savoir quelles revues professionnelles ils préfèrent et pourquoi.

Je suis abonnée à plusieurs publications professionnelles du secteur environnemental (version électronique et papier) en allemand et en anglais : pour l'allemand, EUWID Recycling und Entsorgung et Recycling-Magazin, ainsi que plusieurs lettres d'information en ligne publiées par des organisations environnementales et des associations du secteur. Je lis aussi régulièrement www.letsrecycle.com pour les informations sur la gestion des déchets au Royaume Uni, Waste News et Scrap Magazine pour les évolutions aux États-Unis et regarde la chaîne Environmental News Network pour des informations plus générales dans le domaine de l'environnement. Pour le marché français, je recommande la lecture de Recyclage et Récupération. Autre outil utile, les "Alertes Google Actualités " peuvent être configurées pour vous prévenir par courriel chaque fois que des articles contenant une combinaison de mots clés sont publiés sur Google Actualités.

Si vous n'êtes pas tout à fait convaincu(e) de la nécessité de ces lectures, voici

\section{Cinq bonnes raisons de lire des revues professionnelles}

\section{1) Rester à jour}

La motivation principale de l'abonnement à des revues professionnelles est de rester à jour en suivant les évolutions dans le secteur de la gestion et du recyclage des déchets. Tout traducteur ayant choisi un marché de niche doit se tenir au courant de ce qui se passe dans le domaine concerné. Lorsque la société X m'appelle à propos d'un projet, je conforte mon image de professionnelle fiable en leur montrant, par exemple, que je suis au courant de leur acquisition très récente de la société $Y$.

\section{2) Trouver des clients potentiels}

Autre usage judicieux des revues professionnelles : elles peuvent représenter une réserve de clients potentiels. L'une des publications hebdomadaires que je reçois possède une page regroupant toutes les entreprises mentionnées dans ses articles, avec leur adresse (concrète et virtuelle). Je pratique la lecture avec deux surligneurs - un jaune pour les entreprises qui pourraient avoir des besoins de traduction et un vert pour la terminologie (voir point 4 ci-dessous). J'entre les références dans un fichier Excel aux fins de contact avec certains ou encore pour entreprendre une campagne ciblée à l'approche des salons spécialisés. 


\section{3) Faire de la publicité}

Pour rester sur ce sujet, il ne faut pas oublier que les lecteurs de ces revues constituent un public idéal pour la promotion de vos services. Pourquoi ne pas interroger l'éditeur pour connaître les tarifs d'un encart publicitaire de dimensions raisonnables et voir si cela peut entrer dans votre budget publicité ? N'hésitez pas à le choisir en couleurs si vous pouvez vous le permettre. Sachez qu'une traduction de volume moyen peut suffire à compenser le coût d'une publicité annuelle. Ne faites pas les choses à moitié : consultez éventuellement un professionnel compétent pour concevoir une annonce qui corresponde à l'image que vous souhaitez donner.

4) Effectuer un travail de veille terminologique

Ces revues constituent en outre une excellente source de vocabulaire, ce qui en soi justifie de les lire régulièrement. Les articles écrits par des experts m'ont aidée plus d'une fois à résoudre un casse-tête terminologique. En outre, certains journalistes travaillant pour ces publications se sont volontiers prêtés au remue-méninges pour trouver des équivalents possibles à des mots nouveaux n'ayant pas encore leur version anglaise.

Lorsque je lis mes revues allemandes, surligneur vert en main, je prends le temps de parcourir un maximum d'articles à la recherche de termes liés à l'environnement que je ne connais pas encore. Je les rassemble dans un simple fichier Excel avec trois colonnes : " allemand ", " anglais " et " source/autre information ". Lorsque j'ai un peu de temps, ou lors d'une pause dans mon travail, je reprends le fichier pour faire des recherches sur les termes et leur traduction possible en anglais. Le fichier reste ouvert et j'y ajoute d'autres mots rencontrés en traduisant pour lesquels je ferai une recherche ultérieurement. Je fais de même pour le vocabulaire anglais, mais dans un second temps.

J'ai commencé récemment un fichier tout anglais avec plusieurs feuilles distinctes pour les différents thèmes - changement climatique, récupération / élimination des déchets, déchets électriques et électroniques, exportation de déchets, etc. Chaque feuille comporte des indications sur les législations et initiatives internationales ainsi que des liens vers les textes de loi (qui apparaissent dans nombre de mes textes) et autres précisions sur le secteur. Ainsi, la feuille " Déchets électriques et électroniques " contient des informations sur les différentes catégories d'appareils et les matériaux posant un problème, la directive de l'UE concernant ces déchets, les législations britannique et allemande mettant en œuvre cette directive, les associations du secteur, les programmes de récupération en Europe et l'approche américaine (ou son absence) quant à la gestion des déchets. La collecte de ces informations me permet de rester à jour face aux évolutions de ce domaine et je gagne donc du temps en recherche terminologique durant la traduction.

5) Travailler pour les revues spécialisées

Les revues professionnelles ont elles aussi besoin de traducteurs et de correcteurs - deux des revues auxquelles je suis abonnée sont publiées en allemand et en anglais. II peut donc être 
utile de prendre contact avec la rédaction pour proposer ses services de traduction ou de correction. En tant que fournisseur, vous pourriez alors négocier un tarif préférentiel pour votre encart publicitaire. Je travaille pour une revue professionnelle qui m'a même offert l'abonnement à sa version allemande, en bonus !

\section{Exercer comme traducteur spécialisé dans l'environnement}

Rares sont les organisations dédiées à la protection de l'environnement qui possèdent un budget traduction ; le traducteur spécialisé ne doit pas hésiter à étendre ses recherches pour élargir sa clientèle.

Avant de passer de la situation de traductrice salariée à celle de traductrice indépendante, j'ai pris le temps de réfléchir aux clients ayant des besoins de traduction dans le domaine de l'environnement (plus précisément, dans mon cas, la gestion des déchets) et j'ai vérifié qu'ils étaient susceptibles d'accepter mes tarifs. Ma réflexion a débouché sur les conclusions cidessous.

\section{Catégories de clients potentiels}

\section{1) Acteurs importants hors domaine de l'environnement}

J'ai constaté que les rapports sur la responsabilité sociale des entreprises (RSE) sont manifestement très en vogue depuis quelque temps en termes de traduction spécialisée dans l'environnement - une tendance apparue vers la fin des années 1990 avec la publication par les entreprises de rapports sur l'environnement vantant le nombre de litres d'eau, de tonnes de déchets et d'émissions de gaz à effet de serre économisés au cours de l'année écoulée. Ces documents sont désormais remplacés par les rapports RSE, publiés tous les ans avec le rapport annuel classique. Le champ de ces rapports s'est en outre élargi progressivement pour englober ce que l'on appelle le triple bilan (population, planète, profit). Autrement dit, les entreprises sont jugées non seulement sur leurs résultats financiers, mais aussi sur leurs performances sociales et leur impact sur l'environnement. Ces rapports RSE ne sont pas réservés aux entreprises fournissant des services liés à l'environnement. Cependant, il est assez difficile pour un indépendant de se voir confier ce type de documents. D'après mon expérience, beaucoup de grandes entreprises sous-traitent ces projets auprès d'agences de traduction ou de groupements de traducteurs, en raison du nombre de langues et du volume des documents concernés.

\section{2) Fournisseurs de services dans le domaine de l'environnement}

Outre les rapports RSE, les multinationales fournissant des services dans le domaine de l'environnement ont de nombreux documents à traduire. N'oublions pas que beaucoup de PME cherchent à grandir hors de leurs frontières et doivent donc faire traduire un grand nombre 
de brochures, rapports annuels et divers documents. Outre les communiqués de presse et autres documents de communication d'entreprise, ces PME ont généralement des documents internes à diffuser auprès de leurs employés dans leurs différentes succursales, ce qui représente un flux de travail régulier une fois que vous avez un pied dans la place.

\section{3) Sociétés de conseil, revues professionnelles et instituts de recherche}

Les sociétés de conseil et les instituts de recherche investissent beaucoup d'argent dans les projets et souhaitent donc assurer une diffusion aussi large que possible de leurs résultats ; cela peut aller des communiqués de presse et sites internet ordinaires jusqu'aux études hautement spécialisées des chercheurs. Vous pouvez aussi vérifier quelles sont les revues professionnelles importantes dans votre domaine de spécialité sur le marché de l'environnement et s'il en existe des versions étrangères (ne serait-ce qu'aux fins de recherches terminologiques).

\section{4) Organismes publics}

Les administrations nationales et locales constituent aussi un gisement de travail assez varié, allant des accords multinationaux sur l'environnement aux brochures municipales destinées aux minorités. II existe autant d'approches pour décrocher des contrats avec des organismes publics que de tarifs pratiqués par ces derniers. Ainsi, certains organismes de la haute fonction publique, surtout en Europe, publient des appels d'offre annuels ou bisannuels, tandis que d'autres sous-traitent auprès d'agences de traduction. Le plus simple est de contacter le bureau des relations publiques pour connaître la procédure de recrutement des traducteurs.

\section{5) Agences de traduction}

II ne faut pas oublier les agences. En fait, elles ne représentent qu'un très faible pourcentage de mes revenus car je préfère, pour plusieurs raisons, travailler avec des clients directs. Je ne travaille pour elles que très rarement sur des projets non environnementaux et j'ai précisé à la plupart des agences pour lesquelles j'ai travaillé que je suis désormais spécialisée dans l'environnement et que je ne me disperse plus dans divers domaines.

Lorsque les cibles sont clairement définies, il est temps d'agir. Après avoir pris note de la date des appels d'offre à venir d'organismes publics, j'ai contacté des agences de traduction plus importantes pour leur faire connaître ma spécialité. La recherche de clients directs a montré que la plupart des grandes entreprises avaient déjà leurs traducteurs ou agences ; la meilleure solution était donc de démarcher les entreprises susceptibles de se développer.

\section{Démarches utiles}

1) S'abonner et lire les publications spécialisées du secteur pour connaître les entreprises cherchant à se développer ou celles qui ont décidé d'ouvrir des succursales à l'étranger et les 
contacter. Mentionner la source de votre information quant à leurs projets, confortant ainsi auprès du client l'image du professionnel qui se tient à jour des évolutions du secteur.

2) Assister aux salons professionnels, en lien avec le point précédent. Lorsqu'un client potentiel peut associer un visage à votre nom, vous avez plus de chances de gagner (et, plus important encore, de garder) ses projets et sa confiance. Évitez de harceler les entreprises le premier jour du salon, attendez plutôt un moment plus calme, sans public sur le stand et n'en faites pas trop. Abordez un sujet d'actualité dans le secteur (une fusion majeure, une nouvelle législation, etc.) ou posez une question de terminologie qui vous semble épineuse. De retour chez vous, envoyez un bref message remerciant la personne pour son accueil et mentionnez votre disponibilité professionnelle.

3) Faire connaître son nom. L'une des meilleures solutions - je lui dois la plupart de mes clients actuels : le bouche à oreille. Veillez à ce que vos confrères connaissent votre spécialisation et donnez leurs noms lorsque vous ne vous sentez pas compétent(e) pour une traduction : ils feront de même. Écrivez des articles pour les publications professionnelles, présentez des communications, créez un blog, agissez au sein de votre association de traducteurs et distribuez généreusement votre carte à tous ceux que vous croisez et qui seraient susceptibles d'avoir des besoins de traduction dans le secteur de l'environnement. Évidemment, il vous faut un site internet, de préférence dans vos langues source(s) et cible ; vos clients potentiels pourront ainsi vous contacter plus facilement et connaître votre parcours.

Si vous êtes jeune diplômé ou nouveau traducteur, vous pouvez rechercher des traductions bénévoles pour alimenter votre $\mathrm{CV}$ - il va sans dire qu'il vous faut posséder une bonne connaissance des questions environnementales avant de vous lancer dans un projet de traduction, payé ou pas. Fournir un service gratuit n'est pas une raison pour rendre des traductions bâclées. Si vous faites les choses correctement, vous devriez pouvoir obtenir que l'organisme mentionne, sur tout document imprimé, votre nom (traduit par...) et votre site internet. En outre, la recherche terminologique ne pourra que vous être utile. Dans l'idéal, quelqu'un lira un document mentionnant votre nom et, impressionné par votre maîtrise de la terminologie spécialisée ou parce qu'il aura justement besoin d'un traducteur (de préférence pour un travail rémunéré), il vous appellera. Toutes les règles classiques s'appliquent : soyez agréable et professionnel. Informez clairement votre contact qu'il lui faudra accepter une certaine souplesse dans les délais car toute traduction payée aura priorité sur le travail bénévole. II ne devrait pas y avoir de problème - après tout, il bénéficie de vos services gratuitement.

\section{Réduire son empreinte environnementale}

Je ne saurais conclure cet article sans inclure quelques conseils sur la façon dont nous, traducteurs, pouvons faire notre part pour la protection de l'environnement. 
- Limiter sa consommation d'énergie. Éteindre la lumière si l'on quitte une pièce pour plus de quelques minutes. Brancher son matériel de bureau sur une prise multiple à interrupteur pour tout éteindre lorsque l'on s'absente.

- Imprimer en recto-verso.

- Recycler le papier.

- Utiliser du papier sans chlore, recyclé si possible. Les papeteries utilisant le chlore pour blanchir le papier libèrent des produits toxiques susceptibles de polluer l'eau qui sont donc dangereux pour les animaux et les écosystèmes.

- Si possible, réparer son ordinateur pour le faire durer. Après avoir effacé toutes les données d'un ordinateur hors d'usage, vous pouvez en faire don aux écoles, centres sociaux ou organisations non gouvernementales.

info@printtranslations.com

Abigail Dahlberg est traductrice professionnelle depuis plus de dix ans. Titulaire d'un Master de traduction et interprétation de l'Université Heriot-Watt à Édimbourg, elle a d'abord travaillé plusieurs années comme traductrice et journaliste pour une revue professionnelle allemande dans le domaine $d u$ recyclage et de la gestion des déchets avant de créer Print Translations, une société de traduction spécialisée dans ce secteur.

L'article ci-dessus est issu d'un texte initialement publié dans l'ATA Chronicle, revue de l'American Translators Association, qui a été remanié en 2013 par son auteur à l'intention des lecteurs de Traduire et traduit par Hélène Ladjadj. 